\title{
Análisis de confianza de las entidades bancarias, desde una perspectiva de género
}

\author{
GARCÍA COSTA, LAURA \\ Universidad Rey Juan Carlos (Madrid) \\ Correo electrónico: laura.garcia.costa@urjc.es \\ GARCÍA COSTA, BEATRIZ \\ Universidad Rey Juan Carlos (Madrid) \\ Correo electrónico: beatriz.gcosta@urjc.es \\ GÓMEZ MARTÍNEZ, RAÚL \\ Universidad Rey Juan Carlos (Madrid) \\ Correo electrónico: raul.gomez.martinez@urjc.es
}

\begin{abstract}
RESUMEN
El artículo tiene como objetivo, analizar si la toma de decisión para cambiar de banco presenta diferencias significativas entre mujeres y hombres.

Para su realización, se ha utilizado una encuesta como técnica de investigación, distribuida a 209 personas, inspirada en la que realiza la Universidad de Michigan sobre el índice de confianza del consumidor. Como variables explicativas se determinan la situación bancaria actual, la situación de las finanzas en el momento actual, el grado de rentabilidad de los productos bancarios, la situación bancaria retrospectiva y la situación bancaria prospectiva. La metodología de estudio utilizada, se basa en dos modelos econométricos, lineal y no lineal, modelo probit y logit estimado a partir de las encuestas recopiladas. Como principal conclusión se precisa que sí existen diferencias significativas entre mujeres y hombres en la toma de decisión de cambiar o permanecer en su banco.
\end{abstract}

Palabras clave: género, confianza bancaria, lealtad, modelo econométrico, comportamiento.

Clasificación JEL: C01; D14; I31.

MSC2010: 62P20; 35Q91; 91G70.

Artículo recibido el 26 de octubre de 2019 y aceptado el 2 de julio de 2020. 


\title{
Confidence analysis of the banking entities, from a gender perspective
}

\begin{abstract}
The article aims to analyze, if the decision to change the banks present significant differences between women and men.

For the purpose, a general survey distributed to 209 people was used, inspired by the one conducted by the University of Michigan on the consumer confidence index. As explanatory variables, the following are determined: the current banking situation, the current financial situation, the degree of profitability of banking products, the retrospective banking situation and the prospective banking situation. The study methodology used is based on an econometric model estimated logit model from the surveys collected. The main conclusion is that there are significant differences between women and men in the decision to change or remain in their bank being the most conservative and risk-averse women.
\end{abstract}

Keywords: gender, bank confidence, loyalty, econometric model, behavior.

JEL classification: C01; D14; I31.

MSC2010: 62P20; 35Q91; 91G70.

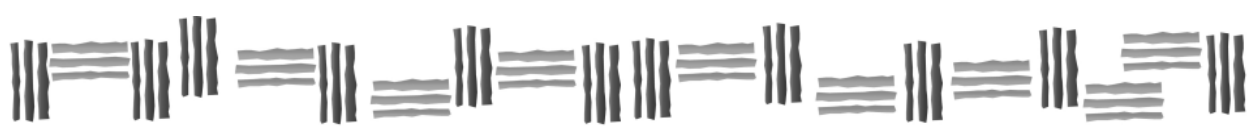




\section{Introducción.}

El sistema económico que vivimos se caracteriza por desigualdades en materia de economía, sociales y comportamentales. En el presente artículo se va a hacer alusión al análisis de desigualdades de comportamiento analizando el género como elemento diferenciador. Por género nos referimos a un concepto con gran relevancia en las ciencias sociales contemporáneas, en la que se explican desigualdades provenientes de roles asignados por la sociedad a los hombres y a las mujeres (Herdoiza, 2015). Las diferencias de comportamiento, entre hombres y mujeres es un tema que ha suscitado distintas opiniones, existiendo diferencias biológicas entre hombres y mujeres determinando que la organización funcional del cerebro y las capacidades mentales de hombres y mujeres presentan diferencias significativas. Las investigaciones en ciencias cognitivas, particularmente neuropsicología, así lo constatan (García, 2003). Se pretende realizar una contribución, que permita identificar roles o comportamientos asociados a mujeres y roles o comportamientos asociados a hombres, en la toma de decisión de permanecer o abandonar una entidad bancaria.

Al identificar que existen diferencias significativas entre hombres y mujeres en elementos comportamentales, se va a analizar la efectividad o no del análisis de decisión de entidades bancarias, mediante unas variables propuestas como son la situación bancaria actual, la situación de las finanzas en el momento actual, el grado de rentabilidad de los productos bancarios, la situación bancaria retrospectiva y la situación bancaria prospective. Se contextualiza en torno a las entidades bancarias, debido a la gran transformación que han sufrido desde el periodo de crisis económica y financiera en la que España se vio inmersa.

Estudiamos si la decisión a cambiar de banco, puede ser explicada mediante las variables propuestas, con el fin de confirmar las diferencias de comportamiento que existen atendiendo a su género. Determinados estudios como el de Lemmon y Portniaguina (2006) demostraron que las encuestas de confianza del consumidor tenían capacidad predictiva sobre la evolución de los mercados bancarios. La realidad del sistema bancario español se ha visto caracterizada por la necesidad de aumentar la vinculación con los clientes. Este punto es considerado uno de los objetivos empresariales más importantes para las entidades financieras (World Retail Banking Report, 2017). Las variables propuestas vienen explicadas a través de una encuesta que replica el Indicador de Confianza del Consumidor (Consumer Confidence Survey, 1967). Esta encuesta se replica teniendo en cuenta la información bancaria.

El objetivo del índice se basa en anticipar los comportamientos de consumo de los ciudadanos, por lo que su construcción se fundamenta principalmente en preguntas sobre las percepciones de los consumidores tanto en su relación con la evolución económica reciente (6 meses), como con respecto a su potencial evolución en el futuro inmediato (6 meses). Tiene su antecedente metodológico en los elaborados por la Universidad de Michigan y The Conference Board (1967) en Estados Unidos.

Harding y He (2016) analizaron cómo influye el estado de ánimo de los inversores respecto a las decisiones de inversión, realizando un ejercicio experimental. Los resultados obtenidos en este experimento mostraron que un estado de ánimo negativo aumenta el nivel de aversión al riesgo; en cambio los inversores inducidos en un estado de ánimo positivo son menos adversos al riesgo. Hirshleifer y Subrahmanyam (1998) proponen una teoría de las reacciones exageradas basadas en sesgos psicológicos. Un exceso de confianza implica autocorrelaciones negativas y exceso de volatilidad; en cambio la autoatribución sesgada determina correlaciones cortas y positivas lo que deriva en ganancias a corto plazo. Niederle y Vesterlund (2007), Eckel y Grossman (2008) promueven la economía experimental para el análisis de diferencias de género, concluyendo diferencias significativas en los comportamientos de género.

Poniendo de manifiesto lo acontecido y desarrollado por los diferentes autores, se establece el objeto de estudio presente, que se basa en analizar si existen diferencias de género en el momento de abandonar o permanecer en la entidad bancaria contextualizado en el paradigma bancario. Este análisis, viene explicado mediante las variables propuestas definidas en: la situación bancaria actual, la situación 
de las finanzas en el momento actual, el grado de rentabilidad de los productos bancarios, la situación bancaria retrospectiva y la situación bancaria prospectiva.

La estructura del artículo considera, en primer lugar, la revisión de las investigaciones realizadas por diversos autores respecto a las diferencias de género y las variables propuestas con el objetivo de comprobar si existen predictores comportamentales de género. En segundo lugar, mostramos el planteamiento de hipótesis, seguido de la metodología y el diseño de la muestra. En la parte final, se exponen los resultados obtenidos y las principales conclusiones.

\section{Marco teórico.}

Se ha demostrado que el género influye en el momento de la toma de decisions y prueba de ello es que en los últimos años se han detectado anomalías en los mercados financieros que llevan a determinar que en los mercados no existe la eficiencia perfecta que tanto se defendió en el pasado siglo. Existen diversos sesgos de comportamiento que demuestran la imperfección de los mercados definiendo una nueva línea de investigación conocida como el comportamiento de las finanzas o "Behavioral Finance". Esta línea de investigación analiza el estudio del comportamiento de las finanzas, basada en los seres humanos, considerados como seres imperfectos en los que se observan diferencias en sus comportamientos y en la toma de decisiones, teniendo como fundamento el conjunto de teorías cognoscitivas y aspectos psicológicos que contextualizan, el porqué y el cómo de sus actos.

En este caso, se pone de manifiesto el género como factor diferenciador en la toma de decisiones debido a que en las entidades bancarias el dinero no representa lo mismo para todos.

En los últimos tiempos, se ha utilizado la economía experimental para el análisis de diferencias de género en un gran número de estudios, encontrando diferencias significativas de comportamiento entre hombres y mujeres. Gneezy y Rustichini (2004) analizaron las brechas de género exponiendo la existencia de diferencias en la equidad del proceso de selección y, en general, sobre la asignación de oportunidades. Charness y Gneezy (2012) plantearon la cuestión, que permitía conocer si los hombres tenían una menor aversión al riesgo que las mujeres, evidenciando como principal conclusión que las mujeres invierten menos $\mathrm{y}$, por lo tanto, tienen una mayor aversión financiera al riesgo que los hombres. Niederle y Vesterlund (2007) expusieron, las diferencias de género estudiando las preferencias de cada uno, determinaron que éstas vienen impulsadas por diferencias en el rendimiento. Eckel y Grossman (2008) revisaron los resultados de las medidas experimentales de aversión al riesgo evidenciando significativos hallazgos en base a las diferencias sistemáticas en el comportamiento de hombres y mujeres. Buchan, Croson y Solnick (2008) consideraron que el género influía en la confianza, la probabilidad de ser confiable y el nivel de confiabilidad, compararon las opciones de hombres y mujeres en el juego de inversión, utilizando los datos de un cuestionario con el fin de comprender las motivaciones de las diferencias de comportamiento. Existen diferencias de género en las actitudes frente al riesgo y estas diferencias dependerán del entorno donde se desenvuelve el individuo (Salazar \& Ospina, 2016).

Estas variaciones de comportamiento financiero de género tienen importantes implicaciones económicas y sociales tanto en el ámbito del consumo, como en el de la inversión y en el del mercado laboral (Blau \& Kahn, 2000; Watson \& McNaughton, 2007).

Gutiérrez y Montiel (2009) pusieron de manifiesto que existen evidencias que afectan a la conciliación de la vida laboral, familiar y personal de hombres y mujeres realizando diferenciación por género. En la toma de decisiones de género, también se atiende a un factor relevante, la lealtad, definida como el compromiso hacia la elección de repetir la compra o ser cliente efectivo de un producto o servicio en el futuro, a pesar de las influencias externas y de los esfuerzos comerciales que influyan potencialmente hacia un comportamiento de cambio (Oliver, Rust \& Varki, 1997). 
Desde el punto de vista de las entidades bancarias se enmarca la intención de recomendar los servicios bancarios a terceras personas, teniendo una actitud favorable hacia la entidad bancaria. Un gran número de autores han estudiado la importancia de la lealtad. Dick y Basu (1994) defienden la lealtad del cliente como un concepto multidimensional que consiste en un comportamiento basado en la actitud.

La lealtad bancaria contempla la actitud hacía la entidad y el comportamiento de seguir utilizando los servicios. Keller (1993) aboga porque la lealtad se presenta cuando la acumulación de actitudes hacia la marca se manifiesta en el comportamiento de compra repetido. Zeithaml, Berry y Parasuraman, (1996) añadieron la intención de recomendación a terceros en la medida de lealtad, entendiendo ésta como un constructo multidimensional. La lealtad es entendida como un comportamiento repetitivo de compra que tienen y que mejora la relación entre ambas partes, clientes y entidades (Bass, 1974; Mick, \& Fournier, 1998).

El valor del cliente unido a indicadores de confianza, permitieron establecer la relación bilateral (Gil-Lafuente, Ortigosa \& Merigó, 2016). Atendiendo a la satisfacción y confianza de los clientes, Rust y Oliver (1994) concibieron que si la satisfacción recibida por parte de un servicio es óptima permitirá al cliente establecer relaciones sólidas con la entidad bancaria y fomentar relaciones a largo plazo mejorando rentabilidades y vinculación de clientes. Shankar, Sultan y Urban, (2002) investigaron acerca de los determinantes y el papel de la confianza del consumidor en el negocio electrónico, examinando las percepciones de confianza del consumidor en un sitio web.

Ranaweera, McDougall y Bansal (2005) consideraron un modelo teórico que capturaba las características principales del sitio web y explicaba cómo la reacción del usuario al sitio web viene determinada por un conjunto de características que podría moderar las percepciones del consumidor de los sitios web, así como los comportamientos posteriores, concluyendo que las percepciones del cliente tienen una gran importancia.

Westbrook y Oliver (1981) centraron su estudio de investigación en la comparación entre los resultados de la experiencia alcanzada y las expectativas previas. El estado psicológico de seguridad produce que el consumidor sea vulnerable a las acciones y resultados de la otra parte.

Mayer, Davis y Schoorman (1995) presentaron una definición de confianza y un modelo de sus antecedentes y resultados, que integraban la investigación de múltiples disciplinas y diferenciaban la confianza de construcciones similares. Rempel, Holmes y Zanna (1985) probaron un modelo teórico de confianza, obteniendo como principal conclusión la existencia de que la confianza disminuía la incertidumbre, la selección adversa, los escenarios de pánico y la sensación de inseguridad.

Las variables explicativas propuestas están definidas por unos ítems propuestos basados en la encuesta Indicador de Confianza del Consumidor (Consumer Confidence Survey, 1967). La variable objetivo es la disposición de cambiar de banco desagregado en género.

La revisión de la literatura pone de manifiesto que el estudio de las variables definidas puede influir en la toma de decisión de las personas a cambiar o permanecer en su banco existiendo diferencias de toma de decisión entre hombres y mujeres. La principal aportación de este estudio se especifica mediante dos modelos econométricos: un modelo lineal planteado en modelo probit y un segundo enfoque no línea planteado en modelo logit. En ambos modelos, se evidencia una aportación concluyente del objeto de estudio planteado. 


\section{Hipótesis.}

Teniendo en cuenta la literatura citada en el epígrafe anterior se pretende analizar, sí con los datos obtenidos en el índice de confianza del consumidor enfocado al sector bancario se determinan diferencias significativas entre hombres y mujeres en el momento de tomar la decisión de permanecer o abandonar su entidad bancaria.

H1: Existe mayor propensión al cambio de banco en situaciones bancarias adversas.

H2: Los hombres son menos fieles a su entidad bancaria.

Los datos replicados a través de una encuesta del índice de confianza del consumidor, enfocado al sector bancario, determina diferencias significativas entre hombres y mujeres en el momento de tomar la decisión de permanecer o abandonar la entidad bancaria.

Atendiendo a las hipótesis planteadas, se realizarán dos análisis, un modelo desde la perspectiva probit y un segundo análisis logit. Sobre la base de los análisis propuestos, se determinará si las variables utilizadas en los modelos son significativas y tienen capacidad de acierto. Se expone el supuesto de que las variables propuestas y la variable endógena "género" tiene impacto en la toma de decisiones de las personas a permanecer o cambiar de entidad bancaria.

\section{Metodología.}

La metodología utilizada para analizar la relación entre el comportamiento de mujeres y hombres, sigue el paradigma planteado de cambiar o permanecer en su entidad bancaria. Realizando mediante dos análisis, un análisis econométrico lineal modelo probit y un segundo análisis modelo no lineal logit. Los resultados se obtienen de una encuesta de elaboración propia que replicará las bases teóricas del índice de confianza del consumidor enfocado al sector bancario (Consumer Confidence Survey, 1967).

Las variables explicativas se detallan en las siguientes:

- Género: Mujer/Hombre

- ¿Cómo describiría la situación actual de los bancos/Entidades Bancarias? ¿Cómo la calificaría Ud.: Muy satisfecho, Satisfecho, Indiferente, Insatisfecho o Muy Insatisfecho?

- ¿Cómo describiría la situación actual de sus finanzas personales? ¿Cómo la calificaría Ud.: Muy satisfecho, Satisfecho, Indiferente, Insatisfecho o Muy Insatisfecho?

- ¿Cómo considera el momento actual, para obtener rentabilidad/beneficio con los servicios que ofrecen los bancos? ¿Cómo la calificaría Ud.: Muy satisfecho, Satisfecho, Indiferente, Insatisfecho o Muy Insatisfecho?

- ¿Diría que financieramente se encuentra mejor, peor o igual que hace un año? ¿Cómo lo calificaría Ud.: Mejor, Igual, Peor?

- ¿Cree que dentro de un año estará financieramente mejor, peor o igual que ahora? ¿Cómo la calificaría Ud.: Mejor, Igual, Peor?

- ¿Está dispuesto a cambiar de banco? Sí/No

Para la primera validación de hipótesis, se construye un modelo de regresión lineal en el que la variable endógena es definida en la siguiente pregunta ¿Estaría Ud. Dispuesto a cambiar de banco? Por favor, responda Sí o No. La respuesta "Sí" significa que sí está dispuesto a cambiar de banco y "No" que Ud. No está dispuesto a cambiar de banco. El modelo se divide haciendo una desagregación por género. 
El primer análisis incluye los resultados obtenidos en la encuesta por el género femenino y el segundo análisis considera los resultados obtenidos por el género masculino. En el modelo se plantea la estructura básica del modelo lineal.

$$
\mathrm{Y}_{\mathrm{t}}=\mathrm{a}+\mathrm{bX}_{\mathrm{t}}+\mathrm{u}_{\mathrm{t}} \ldots \ldots \ldots \mathrm{t}=1,2 \ldots 209
$$

donde:

$\mathrm{Y}_{\mathrm{t}}$ : representa la disposición a cambiar de banco con el tamaño muestral seleccionado

$\alpha$ : es el parámetro independiente del modelo

$\beta:$ es el parámetro dependiente, es la variable explicativa.

La variable $Y_{t}$ es la variable cuyo comportamiento se pretende explicar a través de los predictores $\mathrm{X}_{1}, \mathrm{X}_{2} \ldots \mathrm{X}_{\mathrm{t}}$ siendo las distintas variables que se suponen una gran relevancia como variables explicativas.

Para validar la veracidad de las hipótesis, se realiza un modelo econométrico por Mínimos Cuadrados Ordinarios (MCO), utilizando los datos de la encuesta en términos absolutos. Para comprobar la viabilidad de las regresiones planteadas y eliminar la heterocedasticidad, se utilizará el modelo econométrico por Mínimos Cuadrados Generalizados (MCG).

Para el segundo modelo se construye un modelo logit desagregado por género. El análisis logit es una técnica estadística desarrollada para investigar las relaciones entre una variable binaria y una o más variables independientes. Las variables independientes son las variables propuestas y el criterio de clasificación de la variable dependiente está definido en la siguiente pregunta, ¿Estaría Ud. Dispuesto a cambiar de banco? Por favor responda Sí o No. La respuesta "Sí" significa que sí está dispuesto a cambiar de banco y "No" que Ud. No está dispuesto a cambiar de banco. Siendo 1 para las personas que sí deciden cambiar de banco y 0 para las personas que deciden no cambiar de banco. El modelo logit permite, además de obtener estimaciones de la probabilidad de un suceso, identificar los factores de riesgo que determinan dichas probabilidades, así como la influencia o peso relativo que éstos tienen sobre las mismas.

En el modelo de regresión logit la variable endógena (Yi) es en todos los casos es:

$\mathrm{Y}_{\mathrm{i}}$ : Disposición de un cliente a cambiar o permanecer en su entidad bancaria:

Sí: cambian de banco (1)

No: cambian de banco (0)

El modelo de regresión Logit viene expresado en la siguiente ecuación:

$$
\mathrm{Y}_{\mathrm{i}}=(Z i)+u i, i=1, \ldots, t .
$$

donde $Z_{\mathrm{i}}=\beta_{1}+\beta_{2} \mathrm{X}_{2} \mathrm{i}+\ldots \ldots \beta_{\mathrm{k}} \mathrm{X}_{\mathrm{ki}}$ dados los valores de las variables independientes, $\mathrm{x}_{2} \ldots \ldots \mathrm{x}_{\mathrm{k}}$ probabilidades de que la variable dependiente tome los valores 1 y 0 .

Los regresores en ambos modelos vienen definidos en las siguientes variables:

- SIT BANCARIA ACTUAL: Rango de datos de la situación bancaria actual ponderados en la valoración de 0 a 5 , siendo 0 una situación muy insatisfactoria y 5 una situación muy satisfactoria. 
- SIT FINANZAS ACTUAL: Rango de datos de la situación financiera actual ponderados en la valoración de 0 a 5 , siendo 0 una situación muy insatisfactoria y 5 una situación muy satisfactoria.

- GRADO DE RENTABILIDAD DE SUS PRODUCTOS BANCARIOS: Rango de datos del grado de rentabilidad de sus productos bancarios actual ponderados en la valoración de 0 a 5 , siendo 0 una situación muy insatisfactoria y 5 una situación muy satisfactoria.

- SIT BANCARIA REPROSPECTIVA: Rango de datos de la situación financiera actual ponderados en la valoración de 0 a 3 , siendo 0 una situación muy insatisfactoria y 3 una situación muy satisfactoria.

- SIT BANCARIA PROSPECTIVA: Rango de datos de la situación financiera actual ponderados en la valoración de 0 a 3 , siendo 0 una situación muy insatisfactoria y 3 una situación muy satisfactoria.

Los datos han sido extraídos a través de una encuesta.

Se plantea un modelo subdividido en dos grupos: el primero incluye los resultados obtenidos en la encuesta por el género femenino y el segundo modelo considera los resultados obtenidos en el género masculino. La estimación en modelos logit se realiza mediante el método de Máxima Verosimilitud (MV).

Se aceptará la hipótesis 1 cuando al menos existan 3 variables explicativas en ambos modelos planteados (SIT BANCARIA ACTUAL, SIT FINANZAS ACTUAL, GRADO DE RENTABILIDAD DE SUS PRODUCTOS BANCARIOS, SIT BANCARIA REPROSPECTIVA, SIT BANCARIA PROSPECTIVA) que determinen que la proclividad al cambio de banco es mayor en situación adversas.

Se aceptará la hipótesis 2 cuando se afirme según los modelos planteados, que las variables explicativas planteadas en ambos modelos (SIT BANCARIA ACTUAL, SIT FINANZAS ACTUAL, GRADO DE RENTABILIDAD DE SUS PRODUCTOS BANCARIOS, SIT BANCARIA REPROSPECTIVA, SIT BANCARIA PROSPECTIVA) muestren variables más significativas en el caso del género masculino al cambio de entidad bancaria que en el género femenino. Con esta afirmación, se precisa que los hombres son menos fieles a su entidad bancaria.

\section{Diseño de la muestra.}

La base de datos está constituida por las valoraciones obtenidas a través del método de elaboración de una encuesta. La encuesta planteada replica el Indicador de Confianza del Consumidor (Consumer Confidence Survey, 1967), enfocado a clientes de la banca española. En España existe una limitación de datos, debido a que no se dispone de ningún índice, ni ningún indicador de referencia que mida la disposición de un cliente a permanecer o abandonar una entidad bancaria.

El formulario web consta de un total de 15 preguntas, de las que 7 de ellas son las utilizadas en el presente studio; estas cuestiones son de tipo Likert basadas en la base metodológica del Indicador de Sentimiento del Consumidor. Este indicador procede del Índice del Sentimiento del Consumidor elaborado por la Universidad de Michigan y The Conference Board en Estados Unidos. El Indicador de Sentimiento del Consumidor es un indicador que trata de medir la Confianza del Consumidor a largo plazo. En Estados Unidos se refieren a este indicador con las siglas MCSI o como Consumer Sentiment Index o simplemente Consumer Sentiment. Las cuestiones planteadas se presentan en la escala graduada de Likert (1932) que permiten al encuestado posicionarse en determinados ítems que van del 1 al 5, donde el número 1 representa nada satisfecho con su entidad bancaria y 5 muy satisfecho con su entidad bancaria. La escala de tipo Likert es una escala psicométrica comúnmente utilizada en cuestionarios y es la escala de uso más amplio en encuestas para la investigación. 
Las preguntas seleccionadas son cinco que muestran la réplica del Índice del sentimiento del consumidor y dos adicionales para determinar el género del encuestado/a y la variable objetivo disposición a cambiar de banco. El cuestionario se realizó en el año 2018 y 2019, destacando el carácter voluntario y anónimo de las muestras. El canal de comunicación se realizó mediante redes sociales con una consecución de más de 20.000 visualizaciones. En total la muestra final ha estado compuesta por 209 observaciones. El cuestionario se presentó en Google drive y se publicó en distintas plataformas sociales (LinkedIn, Facebook, Whatsapp). Se decidió compartir en redes sociales por la accesibilidad y simplicidad para obtener información y conseguir impacto en un periodo corto de tiempo. Los datos fueron sometidos a un análisis de confiabilidad y consistencia. Para medir la fiabilidad de los datos se utilizó el estadístico Alfa o modelo de consistencia interna de Cronbach, que mostró coeficientes superiores a $0.80(0.878)$ en la escala analizada. Por esta razón se considera que la escala de datos es aceptable y tienen una consistencia fuerte. Se extrajo una muestra final de 209, mediante muestreo aleatorio simple.

\section{Resultados.}

Por medio del software econométrico GRETL se han realizado las validaciones para las hipótesis planteadas. La forma de analizar las regresiones planteadas, es mediante dos validaciones.

En un primer lugar, una estimación lineal, modelo probit y, en segundo lugar, una estimación no lineal, modelo logit, siendo la variable dependiente en ambos casos, la disposición de los clientes a cambiar o permanecer en su banco. Para desarrollar ambas validaciones se han creado dos conjuntos de datos, agrupados en grupo mujeres y grupo hombres. Se dispone de los datos absolutos obtenidos en la encuesta que reflejan el comportamiento de las mujeres y el segundo modelo refleja el comportamiento de los hombres respecto a la disposición a cambiar o permanecer en su entidad bancaria.

En el primer caso, se realiza una estimación probit, en la que la variable dependiente será construida por la disposición de las mujeres a cambiar o permanecer en el banco, y las variables explicativas son las ponderaciones de 0 a 5 de las cinco preguntas planteadas; se realiza una estimación por mínimos cuadrados ordinarios (MCO), en la que la variable dependiente será construida por la disposición de las mujeres a cambiar o permanecer en el banco, y las variables explicativas son las ponderaciones de 0 a 5 de las cinco preguntas planteadas.

Tabla 1. Estimación MCO del modelo-Mujeres 122 observaciones

\begin{tabular}{|c|c|c|c|c|c|}
\hline Variable & Coeficiente & Desv. Típica & Estadístico t & valor p & \\
\hline Const & 0,946513 & 0,159603 & 5,9304 & $<0,00001$ & $* * *$ \\
\hline SIT_BANCOS & $-0,0655827$ & 0,0400428 & $-1,6378$ & 0,10417 & \\
\hline SIT_FINANZAS & 0,0319199 & 0,0403778 & 0,7905 & 0,43083 & \\
\hline RENTABILIDAD & 0,00558104 & 0,0384961 & 0,1450 & 0,88498 & \\
\hline SIT_BANK RETRO & $-0,000581164$ & 0,0586061 & $-0,0099$ & 0,99210 & \\
\hline SIT_BANK_PROSPE & $-0,0165418$ & 0,0553642 & $-0,2988$ & 0,76564 & \\
\hline
\end{tabular}

Fuente: Elaboración propia.

En la Tabla 1 se contemplan los estadísticos descriptivos de las regresiones realizadas en datos absolutos. En el análisis econométrico realizado, encontramos que el parámetro $\beta$ relaciona las 
valoraciones obtenidas en la encuesta y utilizamos cómo variable endógena la disposición a cambiar de banco.

A partir de los datos obtenidos, se concluye más que a la significación del modelo, se validan las hipótesis haciendo alusión a las tendencias de las mismas. Se confirma que en el $60 \%$ de los casos, los resultados son más determinantes en la situación bancaria actual, situación bancaria prospectiva y retrospectiva cuando existe una mayor inestabilidad; la disposición de una mujer cliente de la banca española incide de forma negativa determinando que se incremente el número de mujeres dispuestas a abandonar su banco.

Para eliminar la autocorrelación existente y heteroscedasticidad en las variables estudiadas, se va a plantear el modelo por mínimos cuadrados generalizados (MCG).

Los estadísticos obtenidos por mínimos cuadrados generalizados, confirman que en 3 de los 5 casos analizados la disposición de las mujeres a cambiar o permanecer en banco será mayor cuando nos encontremos ante una situación de inestabilidad bancaria, obteniendo una tendencia significativa al 95\% ante la situación bancaria actual.

Tabla 2. Estimación MCG del modelo-Mujeres 122 observaciones.

\begin{tabular}{|l|c|c|c|c|l|}
\hline \multicolumn{1}{|c|}{ Variable } & Coeficiente & Desv. típica & Estadístico t & valor p & \\
\hline Const & 1,0512 & 0,0950136 & 11,0636 & $<0,00001$ & $* * *$ \\
\hline SIT_BANCOS & $-0,0822634$ & 0,0325263 & $-2,5291$ & 0,01278 & $* *$ \\
\hline SIT_FINANZAS & 0,00458458 & 0,0284493 & 0,1611 & 0,87226 & \\
\hline RENTABILIDAD_ & 0,0050325 & 0,0305626 & 0,1647 & 0,86950 & \\
\hline SIT_BANK_RETRO & $-0,0400991$ & 0,0379442 & 1,0568 & 0,29280 & \\
\hline SIT_BANK_PROSPE & $-0,0424952$ & 0,032351 & $-1,3136$ & 0,19158 & \\
\hline
\end{tabular}

* Intervalo de confianza del $90 \%$.

** Intervalo de confianza del 95\%.

$* * *$ Intervalo de confianza del $99 \%$.

Fuente: Elaboración propia.

Posteriormente, se realiza una estimación por mínimos cuadrados ordinarios, en la que la variable dependiente será construida por la disposición de los hombres a cambiar o permanecer en el banco, y las variables explicativas son las ponderaciones de 0 a 5 de las cinco preguntas planteadas.

En la Tabla 3 se contemplan los estadísticos descriptivos de las regresiones realizadas en datos absolutos. En el análisis econométrico realizado, se establece que el parámetro $\beta$ relaciona las valoraciones obtenidas en la encuesta del género masculino.

Teniendo en cuenta el modelo planteado, se confirma que cuando aplicamos el análisis econométrico obtenemos valores significativos al $99 \%$ en la pregunta de que los hombres deciden cambiar de banco según la situación actual de los bancos, al 90\% de significación los hombres estarían dispuestos a cambiar de banco según la situación de sus finanzas y a un $99 \%$ de significación los hombres estarían dispuestos a cambiar de entidad bancaria si la situación bancaria futura es más inestable. Se considera que el modelo propuesto presenta variables significativas, confirmando que en 3 de los 5 casos planteados se muestra una tendencia negativa, cuando influye la situación bancaria, la situación de las finanzas y la situación bancaria retrospectiva, provoca una mayor tendencia a abandonar su entidad bancaria. 
Tabla 3. Estimación MCO del Modelo-Hombres observaciones 1-87.

\begin{tabular}{|l|c|c|c|c|l|}
\hline \multicolumn{1}{|c|}{ Variable } & Coeficiente & Desv. Típica & Estadístico t & valor p & \\
\hline Const & 0,987259 & 0,227417 & 4,3412 & 0,00004 & $* * *$ \\
\hline SIT_BANCOS & $-0,169641$ & 0,0469417 & $-3,6139$ & 0,00052 & $* * *$ \\
\hline SIT_FINANZAS & $-0,0968027$ & 0,0569679 & $-1,6993$ & 0,09311 & $*$ \\
\hline RENTABILIDAD & 0,0105618 & 0,0485897 & 0,2174 & 0,82847 & \\
\hline SIT_BANK_RETRO & $-0,0395064$ & 0,0777858 & $-0,5079$ & 0,61291 & \\
\hline SIT_BANK PROSPE & 0,237801 & 0,0740701 & 3,2105 & 0,00190 & $* * *$ \\
\hline
\end{tabular}

* Intervalo de confianza del 90\%.

** Intervalo de confianza del $95 \%$.

*** Intervalo de confianza del 99\%.

Fuente: Elaboración propia.

Para eliminar la autocorrelación existente y heteroscedasticidad en las variables estudiadas, se plantea el modelo comprendido en mínimos cuadrados generalizados (MCG). En la Tabla 4 se muestran los resultados obtenidos por mínimos cuadrados generalizados, de la disposición del género masculino a cambiar de banco, determinando una mayor disposición afirmativa ante situaciones de inestabilidad bancaria.

Tabla 4. Estimación MCG del modelo-Hombres observaciones 1-87.

\begin{tabular}{|l|c|c|c|c|c|}
\hline \multicolumn{1}{|c|}{ Variable } & Coeficiente & Desv. típica & Estadístico t & valor p & \\
\hline Const & 1,01431 & 0,1066 & 9,5151 & $<0,00001$ & $* * *$ \\
\hline SIT_BANCOS & $-0,101394$ & 0,0303488 & $-3,3409$ & 0,00126 & $* * *$ \\
\hline SIT_FINANZAS & $-0,0409872$ & 0,0293098 & $-1,3984$ & 0,16581 & \\
\hline RENTABILIDAD & 0,0257918 & 0,0281568 & 0,9160 & 0,36238 & \\
\hline SIT_BANK RETRO & $-0,0522828$ & 0,0509199 & $-1,0268$ & 0,30759 & \\
\hline SIT_BANK_PROSPE & 0,100409 & 0,042511 & 2,3620 & 0,02058 & $* *$ \\
\hline
\end{tabular}
** Intervalo de confianza del 90\%.
*** Intervalo de confianza del 95\%.

Fuente: Elaboración propia.

Según los datos obtenidos por mínimos cuadrados generalizados, se confirma que en 3 de los 5 casos analizados la disposición de los hombres a cambiar de banco será mayor cuando nos encontremos ante una situación de inestabilidad bancaria, mostrando una tendencia significativa al $99 \%$ ante la situación bancaria actual y un nivel de significación del $95 \%$ atendiendo a la situación bancaria prospectiva.

Con los datos analizados por mínimos cuadrados ordinarios y generalizados se determina que, con las variables propuestas, tanto en género femenino como masculino, existe mayor propensión al cambio de banco ante situaciones bancarias adversas, por tanto, se aceptaría la hipótesis H1: Existe mayor propensión al cambio de banco en situaciones bancarias adversas. A la luz de los datos obtenidos con el modelo probit, se establece que el género masculino se ve influenciado por un mayor grado de variables explicativas y un mayor nivel de significación en el momento de cambiar de banco. Se afirma que los hombres muestran menos fidelidad a su entidad bancaria, en consecuencia, confirmando la hipótesis H2: Los hombres son menos fieles a su entidad bancaria.

En la segunda validación, se realiza una estimación logit, en la que la variable dependiente es constituida, por la disposición a cambiar o permanecer en el banco, y las variables explicativas son las ponderaciones de 0 a 5 de las cinco preguntas planteadas. Se procede a analizar en el primer caso, la muestra de mujeres. 
Tabla 5. Estimaciones logit, relación existente entre la variable objetivo "Disposición a cambiar de banco" y variables explicativas, en el caso de género femenino.

\begin{tabular}{|l|l|l|l|l|}
\hline Variable & Coeficiente & Desv. Típica & Estadístico t & Valor p \\
\hline Const & 2,58537 & 1,19126 & 2,1703 & \\
\hline SIT_BANCOS & $-0,44789$ & 0,276162 & $-1,6218$ & $-0,0609112$ \\
\hline SIT FINANZAS & 0,211987 & 0,278362 & 0,7616 & 0,0288294 \\
\hline RENTABILIDAD_ & 0,0283527 & 0,268232 & 0,1057 & 0,00385586 \\
\hline SIT BANK RETRO & $-0,00502179$ & 0,428111 & $-0,0117$ & $-0,000682943$ \\
\hline SIT_BANK_PROSPE & $-0,154183$ & 0,422005 & $-0,3654$ & $-0,0209682$ \\
\hline
\end{tabular}

Fuente: Elaboración propia.

En la Tabla 5 se muestran los estadísticos descriptivos de las regresiones realizadas en datos absolutos. Con los datos analizados en el modelo, se observa que la variable que explica la "SIT_BANCOS" tanto en el momento actual, como en el momento prospectivo y retrospectivo, mantiene una relación inversa con la disposición a permanecer en su banco. Cuando existe una mayor inestabilidad, la disposición de una mujer cliente de la banca española va a replicar de una forma negativa determinando que se incremente el número de mujeres dispuestas a abandonar su banco. En el segundo caso, se realiza una regresión logit del género masculino, en la que la variable dependiente será construida por la disposición de los hombres a cambiar o permanecer en el banco, y las variables explicativas son las ponderaciones de 0 a 5 de las cinco preguntas planteadas.

En la Tabla 6 se contemplan los estadísticos descriptivos de las regresiones realizadas en datos absolutos del género masculino. A la luz de los hallazgos obtenidos, se considera una tendencia significativa al $99 \%$ ante la situación bancaria actual, un nivel de significación del $90 \%$ atendiendo a la situación de las finanzas y un 99\% de significación de la situación bancaria prospectiva.

Tabla 6. Estimaciones logit, relación existente entre la variable objetivo "Disposición a cambiar de banco" y variables explicativas, en el caso de género masculino.

\begin{tabular}{|l|c|c|c|c|l|}
\hline \multicolumn{1}{|c|}{ Variable } & Coeficiente & Desv. típica & Estadístico t & valor p & \\
\hline Const & 3,30425 & 1,67641 & 1,9710 & 0,04872 & $* *$ \\
\hline SIT_BANCOS & $-1,21389$ & 0,382046 & $-3,1773$ & 0,00149 & $* * *$ \\
\hline SIT FINANZAS & $-0,793093$ & 0,462291 & $-1,7156$ & 0,08624 & $*$ \\
\hline RENTABILIDAD & 0,138484 & 0,338952 & 0,4086 & 0,68286 & \\
\hline SIT_BANK RETRO & $-0,20474$ & 0,542613 & $-0,3773$ & 0,70593 & \\
\hline SIT BANK PROSPE & 1,67294 & 0,579884 & 2,8849 & 0,00391 & $* * *$ \\
\hline
\end{tabular}

* Intervalo de confianza del 90\%.

** Intervalo de confianza del $95 \%$.

*** Intervalo de confianza del 99\%.

Fuente: Elaboración propia.

Se observa que la variable que explica la "SIT_BANCOS", "SIT_FINANZAS", "SIT_BANK_RETRO" tanto en el momento actual, como en el momento retrospectivo, mantiene una relación inversa con la disposición a cambiar de banco y la situación de las finanzas tiene una relación inversa. Se confirma teniendo en cuenta las tendencias de las regresiones obtenidas mediante el modelo logit, que los hombres tienen una mayor propensión al cambio de banco. A partir de los datos obtenidos, 
y analizando cada observación del género podemos concluir que más que a la significación del modelo se van a validar las hipótesis haciendo alusión a las tendencias de las mismas. Se confirma que las mujeres y los hombres, género femenino y masculino, tienen una mayor propensión a cambiar de banco, cuando existen situaciones bancarias adversas, confirmando la hipótesis H1: Existe mayor propensión al cambio de banco en situaciones bancarias adversas; cuando existe una mayor inestabilidad, la disposición de un cliente de la banca española incide negativamente determinando que se incremente el número de hombres y mujeres dispuestas a abandonar su banco.

Atendiendo a las diferencias de género, el modelo logit precisa que los hombres tienen mayor predisposición a cambiar de banco que las mujeres, ante esta situación se confirma que se acepta la hipótesis H2: Los hombres son menos fieles a su entidad bancaria.

\section{Discusión y conclusions.}

El objetivo propuesto en esta investigación ha consistido en analizar si existen diferencias de género en el momento de abandonar o permanecer en la entidad bancaria contextualizado en el paradigma bancario. El análisis realizado, ha permitido explicar a través de las variables analizadas cómo la toma de decisiones de permanecer o abandonar la entidad bancaria viene condicionada por las variables explicativas, permitiendo desagregar por género qué sexo es más proclive al cambio.

A la luz de los resultados de la investigación propuesta, se han establecido las siguientes recomendaciones. Como conclusión principal se confirma que la investigación tiene capacidad para afirmar que, ante una situación bancaria adversa, en la que se muestren síntomas de incertidumbre e inestabilidad, la disposición afirmativa a cambiar de banco es mayor indistintamente del género. Tanto hombres como mujeres en ambos casos ante una situación económica convulsa, una situación de incertidumbre bancaria, hombres y mujeres buscan alternativas bancarias.

Según Ruiz-Tagle y Tapia (2012), las mujeres son significativamente más aversas al riesgo que los hombres, pero solo en grupos jóvenes y medios y la brecha en aversión al riesgo tiende a desaparecer en edades próximas a la jubilación.

Según un estudio realizado por Fidelity (2013), las mujeres suelen tener una menor tolerancia al riesgo que los hombres, indicando que el $4 \%$ están dispuestas a invertir una cantidad considerable de dinero para poder obtener una mayor rentabilidad con el riesgo inherente de pérdidas, frente al $15 \%$ de los hombres.

Una encuesta realizada por BlackRock (2013) muestra que las mujeres tienen un mayor porcentaje de dinero en efectivo y están menos dispuestas a arriesgar más para conseguir mayores beneficios.

En el caso de las mujeres, la toma de decisión de cambiar de banco está motivada por la situación bancaria actual, la situación bancaria retrospectiva y prospectiva. Por este motivo, se considera que las mujeres tienen mayor preocupación por el estado actual de los bancos y su futuro y cambiarían ante situaciones de incertidumbre y cambios que se dieran en la entidad bancaria, entendiendo una mayor lealtad por parte de las mujeres hacia su banco. El trabajo de Ricciardi (2008) indica que numerosos estudios experimentales y revisiones basadas en la conducta de los individuos, inciden en la importancia de que las mujeres son más conservadoras que los hombres y tienden a tomar menos riesgos.

Destacar que en el caso de los hombres las variables que influyen en la decisión de cambiar de entidad bancaria se definen en la situación bancaria actual, la situación de las finanzas, y la situación bancaria retrospectiva, y respaldado por un mayor nivel de significación. Todo ello nos muestra una realidad de que los hombres son más proclives al cambio, tienen mayor disposición que las mujeres a 
cambiar de entidad bancaria. Los hombres presentan un comportamiento más influenciable a la hora de tomar la decisión de dónde depositar y dónde invertir sus ahorros.

Este hecho se traduciría en que las mujeres tienen una mayor aversión al riesgo que los hombres y por ello, la toma de decisión de cambiar de banco es más premeditada que en el caso de los hombres que son más proclives al cambio, demostrando menor lealtad a la entidad bancaria.

El sector bancario es un motor principal para la economía española y por ello es necesario impulsar modelos que ayuden a detectar a los bancos qué clientes están dispuestos a permanecer en su entidad, reportándoles beneficios vs oportunidades de futuro y cuáles serán sus clientes potenciales a abandonar su entidad bancaria. Todo ello va a suscitar que el sistema bancario español se vea fortalecido y vuelva a tener una plenitud, satisfacción y confianza.

\section{Referencias}

Bass, F. M. (1974). The theory of stochastic preference and brand switching. Journal of Marketing Research, 11(1), 1-20.

Blackrock Survey (2013). Encuesta global de inversiones sostenibles 2020. Descargado de https://www.blackrock.com/cl/quienes-somos/encuesta-sostenibilidad.

Blau, F.D., \& Kahn, L M. (2000). Gender differences in pay. Journal of Economic Perspectives, 14(4), 75-9

Buchan, N.R., Croson, R.T., \& Solnick, S. (2008). Trust and gender: An examination of behavior and beliefs in the investment game. Journal of Economic Behavior \& Organization, 68(3-4), 466-476.

Charness, G., \& Gneezy, U. (2012). Strong evidence for gender differences in risk taking. Journal of Economic Behavior \& Organization, 83(1), 50-58.

Consumer Confidence Survey (1967). Descargado de https://conferenceboard.org/data/consumerconfidence.cfm

Daniel, K., Hirshleifer, D., \& Subrahmanyam, A. (1998). Investor Psychology and Security Market Under- and Overreactions. The Journal of Finance, 53, 1839-1885. DOI: 10.1111/0022-1082.00077.

Dick, A.S., \& Basu, K. (1994). Customer loyalty: toward an integrated conceptual framework. Journal of the academy of Marketing Science, 22(2), 99-113.

Eckel, C.C., \& Grossman, P.J. (2008). Men, women and risk aversion: experimental evidence. Handbook of Experimental Economics Results, 1, 1061-1073.

Fidelidy Survey (2013). Fidelity Analyst Survey 2021: Counting down the days. Descargado de https://www.fidelityinstitutional.com/en-gb/editorial/fidelity-answers

García, E. (2003). Neuropsicología y género. Revista de la Asociación Española de Neuropsiquiatría, 86, 7-18.

Gil-Lafuente, A. M., Ortigosa, M., \& Merigó, J. M. (2016). Teoría de la incertidumbre aplicada al valor del cliente en situaciones contractuales con intervalos de confianza. Revista de Métodos Cuantitativos para la Economía y la Empresa, 4, 75-97. 
Gneezy, U., \& Rustichini, A. (2004). Gender and competition at a young age. American Economic Review, 94(2), 377-381.

Gutiérrez, O.D.M., \& Montiel, L. (2009). Una cuantificación de las asimetrías por género en materia de conciliación. Investigaciones Regionales, 14, 107-130.

Harding, N., \& He, W. (2016). Investor mood and the determinants of stock prices: an experimental analysis. Accounting \& Finance, 56(2), 445-478.

Herdoiza, M. (2015). Construyendo Igualdad en la Educación Superior. Fundamentos y lineamientos para transversalizar los ejes de igualdad y ambiente. Quito: Senescyt/Unesco.

Keller, K. L. (1993). Conceptualizing, measuring, and managing customer-based brand equity. Journal of Marketing, 57(1), 1-22.

Lemmon, M., \& Portniaguina, E. (2006). Consumer confidence and asset prices: Some empirical evidence. The Review of Financial Studies, 19(4), 1499-1529. Retrieved from http://www.jstor.org/stable/4123480.

Likert, R. (1932). A technique for the measurement of attitudes. New York: The Science Press.

Mayer, R.C., Davis, J.H., \& Schoorman, F.D. (1995). An integrative model of organizational trust. Academy of Management Review, 20(3), 709-734.

Mick, D.G., \& Fournier, S. (1998). Paradoxes of technology: Consumer cognizance, emotions, and coping strategies. Journal of Consumer Research, 25(2), 123-143.

Niederle, M., \& Vesterlund, L. (2007). Do women shy away from competition? Do men compete too much? The Quarterly Journal of Economics, 122(3), 1067-1101.

Oliver, R.L., Rust, R.T., \& Varki, S. (1997). Customer delight: Foundations, findings, and managerial insight. Journal of Retailing, 73(3), 311-336.

Ranaweera, C., McDougall, G., \& Bansal, H. (2005). A model of online customer behavior during the initial transaction: Moderating effects of customer characteristics. Marketing Theory, 5(1), 51-74.

Rempel, J. K., Holmes, J. G., \& Zanna, M.P. (1985). Trust in close relationships. Journal of personality and social psychology, 49(1), 95.

Ricciardi, V. (2008). The Psychology of Risk: The Behavioral Finance Perspective. N.p. Web. DOI: 10.1002/9780470404324.hof002010.

Ruiz-Tagle, V. \& Tapia, P. (2012). Brechas por Género en Aversión al Riesgo. Working Papers wp373, University of Chile, Department of Economics.

Rust, R.T., \& Oliver, R.W. (1994). The death of advertising. Journal of Advertising, 23(4), 71-77.

Salazar, C.S., \& Ospina, D.P. (2016). Género, riesgo y mercado laboral: Evidencia experimental. Escenarios: empresa y territorio, 5(5), 129-150.

Shankar, V., Urban, G. L., \& Sultan, F. (2002). Online trust: A stakeholder perspective, concepts, implications, and future directions. The Journal of Strategic Information Systems, 11(3-4), 325-344. 
Watson, J., \& McNaughton, M. (2007). Gender differences in risk aversion and expected retirement benefits. Financial Analysts Journal, 63(4), 52-62. Retrieved from http://www.jstor.org/stable/4480862.

Westbrook, R. A., \& Oliver, R. L. (1981). Developing better measures of consumer satisfaction: Some preliminary results. Advances in Consumer Research, 16, 94-99.

World Retail Banking Report (2017). Informe Anual 2017. Descargado de https://worldretailbankingreport.com/wpcontent/uploads/sites/3/2018/09/World_Retail_Banking_Report_2017.pdf

Zeithaml, V.A., Berry, L.L., \& Parasuraman, A. (1996). The behavioral consequences of service quality. Journal of Marketing, 60(2), 31-46. 